\title{
Spherical Aberration Corrections for an Electrostatic Gridded Lens
}

\author{
Alexander Pikin

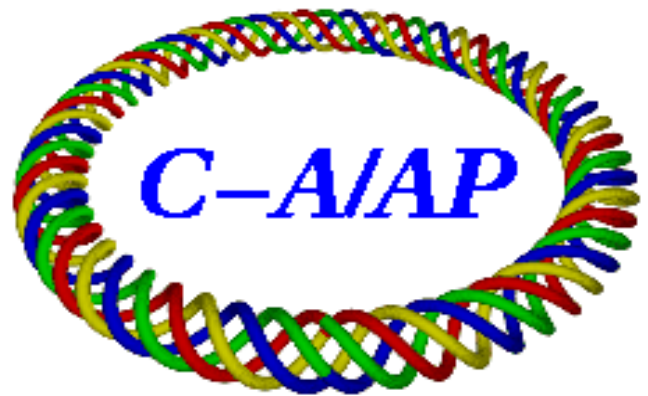 \\ Collider-Accelerator Department \\ Brookhaven National Laboratory \\ Upton, NY 11973
}

Notice: This document has been authorized by employees of Brookhaven Science Associates, LLC under Contract No. DE-AC02-98CH10886 with the U.S. Department of Energy. The United States Government retains a non-exclusive, paidup, irrevocable, world-wide license to publish or reproduce the published form of this document, or allow others to do so, for United States Government purposes. 


\title{
Spherical aberration corrections for an electrostatic gridded lens
}

\author{
Alexander Pikin
}

\begin{abstract}
Two methods of spherical aberration corrections of an electrostatic gridded lens have been studied with ray-tracing simulations. Both methods are based on modifying electrostatic field on the periphery of the lens. In a simplest case such modification is done by extending the part of the grid support on its radial periphery in axial direction. In alternative method the electric field on the radial periphery of the lens is modified by applying an optimum voltage on an electrically isolated correcting electrode. It was demonstrated, that for a given focal length the voltage on this lens can be optimized for minimum aberration. The performance of lenses is presented as a lens contribution to the beam RMS normalized emittance.
\end{abstract}

\section{Introduction}

The gridded lens is a frequent lens of choice in a beam transport tasks with space-charge dominated ion beams where beams fill a significant fraction of ID of optical elements and minimum spherical aberration is required. There is a little alternative to the gridded lens in cases where correction by either focusing or defocusing of the charged particle beams is needed. Like other electrostatic lenses the gridded lens is not free of spherical aberrations caused primarily by different focusing properties of the electric field produced by lens electrodes for ions on the different radii. Since the main contributor of the emittance growth in a beam line is spherical aberration of the beam optics (primarily lenses), it is important to reduce this effect for a typical case when the ion beam fills significant fraction of the lens aperture. Interesting idea of correcting the lens aberration by forming the foils/meshes with simple spherical shape is presented in ${ }^{1,2}$ and with more complex shape, which follows the shape of equipotential needed for virtually free of aberrations focusing ${ }^{3}$. The goal of this work is to study methods of spherical aberration reduction for a gridded lens using ray-tracing simulations.

\section{Methods of aberration correction}

We consider a simple one-grid lens with two cylindrical rings on both sides of the grid inside a cylindrically-symmetric grounded pipe of larger ID (Fig. 1). The ID of these rings defines the opening of the lens and therefore constitutes the lens aperture. 


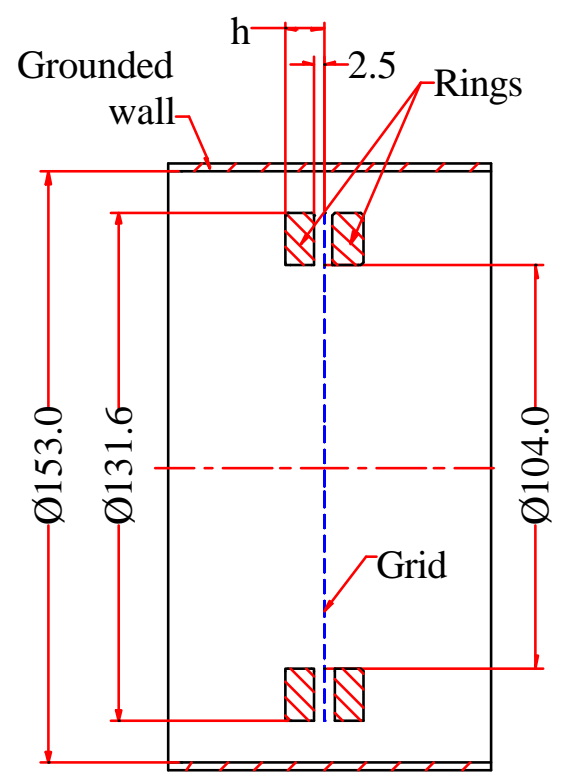

Fig. 1. Schematic of the gridded lens in a pipe used for ray-tracing simulations.

\subsection{Gridded lens with rings at the same potential as the grid (Unipotential lens)}

The symmetrical rings on the periphery of the grid are needed for the grid mount and can be used for the modification of the electrostatic field in this region. In a simple case when these rings have the same potential as the grid, the peripheral electrostatic field can be modified (by making the equipotentials on the radial periphery less steep) by extending these rings in axial direction. The main dimensions in this model reflect the actual dimensions of the lens built in a 2-sided ConFlat vacuum flange for use in a 6" vacuum pipe.

The optical simulations have been done for dimensions of the lens indicated in Fig. 1 with program TRAK ${ }^{4} .500$ trajectories parallel to the axis with energy of $17.5 \mathrm{keV} /$ charge randomly distributed over the circular cross-section with uniform average density and with zero current have been used, so the space charge effect was not considered. Since the initial emittance of the beam is zero, the final emittance can be interpreted as the emittance contribution by the lens or the acquired emittance.

We studied effect of the ring length on the focusing strength of the lens and on the acquired emittance for a fixed gap between grid and rings of $2.5 \mathrm{~mm}$. The focal distance here is defined as a distance from the grid to the Z-position of intersection the trajectory with the longitudinal axis, averaged over all trajectories.

The results of these simulations for a case when both rings are electrically connected to the grid (unipotential lens) are presented in Fig. 2. 


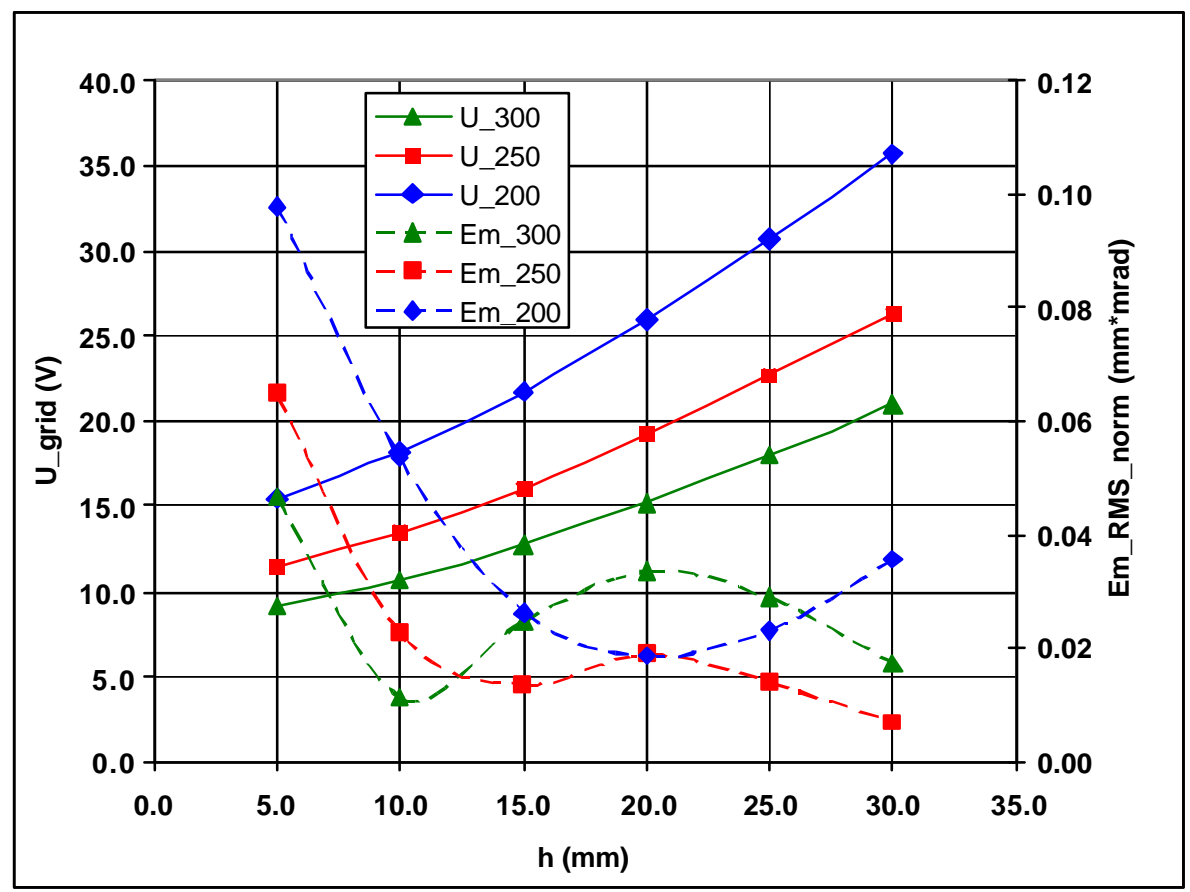

Fig. 2. Dependences of the required voltage on the grid (solid lines) and the acquired RMS normalized emittance of the ion beam (broken lines) on the length of rings $\mathrm{h}$ for fixed focal distances $300 \mathrm{~mm}, 250 \mathrm{~mm}$ and $200 \mathrm{~mm}$. The ion beam radius is $40 \mathrm{~mm}$, ion energy is 17.5 $\mathrm{keV} /$ charge.

One can see that with increased length of the ring it takes higher lens voltage to get the same focal distance. The dependence of emittance growth for a given focal length on the length of the ring $\mathrm{h}$ has minimum for each focal length. This me ans that any fixed length of the rings is optimal (has minimum emittance contribution) only for one focal length. For a different focal length the lens aberration will be larger.

\subsection{Gridded lens with isolated correcting electrode}

It seems attractive to make a lens with spherical aberration correction adaptive for different focal lengths. The solution can be using rings electrically isolated of the grid. With the same potential on these rings this couple of rings can be called a correcting electrode. Such configuration of electrodes can modify the electrostatic field similar to what does different ring length in a case above by applying to the correcting electrode voltage, which is different from the grid voltage.

The model for simulations of this lens concept is similar to Fig. 1, only both rings have the same potential and are isolated from both the grid and the ground and have a fixed length $\mathrm{h}=10$ $\mathrm{mm}$ and a fixed gap between grid and rings of $2.5 \mathrm{~mm}$. For each voltage on the correcting electrode a voltage on the grid was found, which is needed for focusing the ion beam on a fixed distance from the grid $(150 \mathrm{~mm}, 200 \mathrm{~mm}$ and $250 \mathrm{~mm})$ - similar to finding the grid voltage for each grid length in a case above.

The results of simulations are presented in Fig. 3 . 


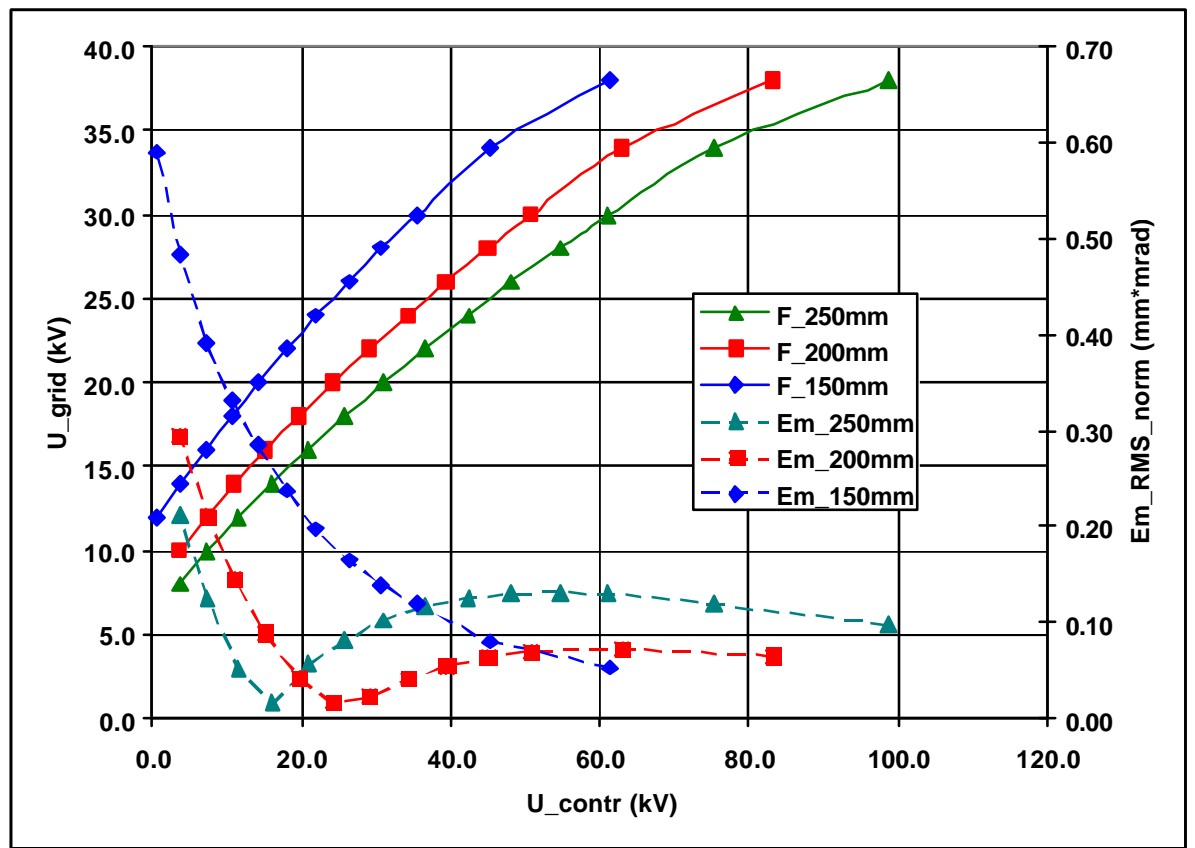

Fig. 3. Dependences of the required voltage on the grid (solid lines) and the acquired RMS normalized emittance of the ion beam (broken lines) on a correcting electrode voltage for fixed focal distances $250 \mathrm{~mm}$ and $200 \mathrm{~mm} 150 \mathrm{~mm}$. The ion beam radius is $40 \mathrm{~mm}$, ion energy is 17.5 $\mathrm{keV} / \mathrm{charge}$.

Both plots (Fig. 2 and Fig.3) look similar, which indicates that the effect of increased correcting electrode voltage is very similar to the effect of increased length of the rings held at the potential of the grid.

The increased voltage on the correcting electrode results in a reducing focusing ability of the electric field on the periphery to the larger extent, then for a near-axis field and this correction has a result of reducing acquired emittance (or spherical aberrations). Decreasing focusing strength on the periphery with further increase of voltage on the correcting electrode has effect of insufficient focusing of the peripheral trajectories (Fig. 5B), which gives raise of the emittance.

The insufficient voltage on the correcting electrode compare to what is needed for optimal focusing results in overfocusing of peripheral trajectories compare to near-axis trajectories (Fig. 4 A).

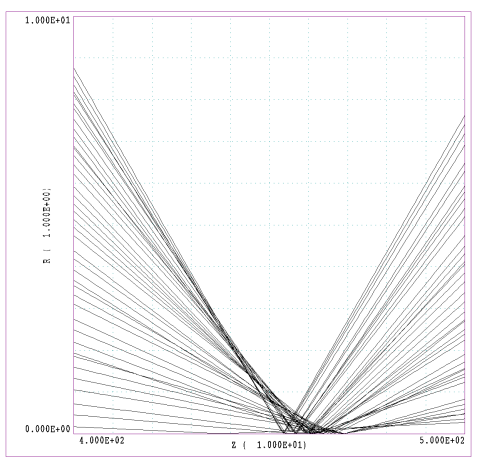

A

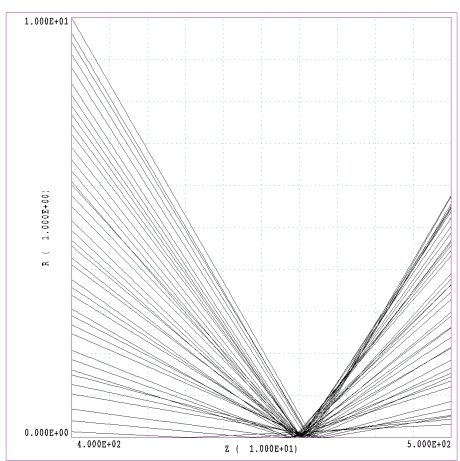

B

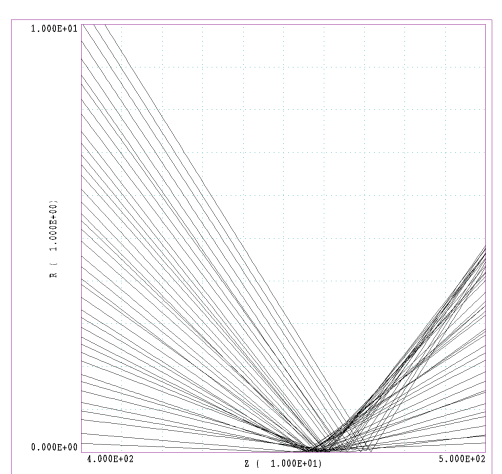

C 
Fig. 4. Trajectory plots at focal distance $250 \mathrm{~mm}$ for overfocusing conditions of peripheral trajectories $\left(A: U_{\text {grid }}=12 \mathrm{kV}, U_{\text {contr }}=11.59 \mathrm{kV}\right)$, for optimal focusing $\left(B: U_{\text {grid }}=14 \mathrm{kV}, U_{\text {contr }}=\right.$ $16.21 \mathrm{kV})$ and for underfocusing conditions $\left(\mathrm{C}: \mathrm{U}_{\text {grid }}=16 \mathrm{kV}, \mathrm{U}_{\text {contr }}=21.06 \mathrm{kV}\right)$

The dependence of acquired emittance on the beam radius for lens optimized for focal distance $F_{\text {focal }}=202 \mathrm{~mm}$ is presented in Fig. 5. With increased beam diameter the acquired emittance is larger, but this dependence is not very steep and in the range of beam radii $r_{\text {beam }}=5-40 \mathrm{~mm}$ it can be fit with a linear function

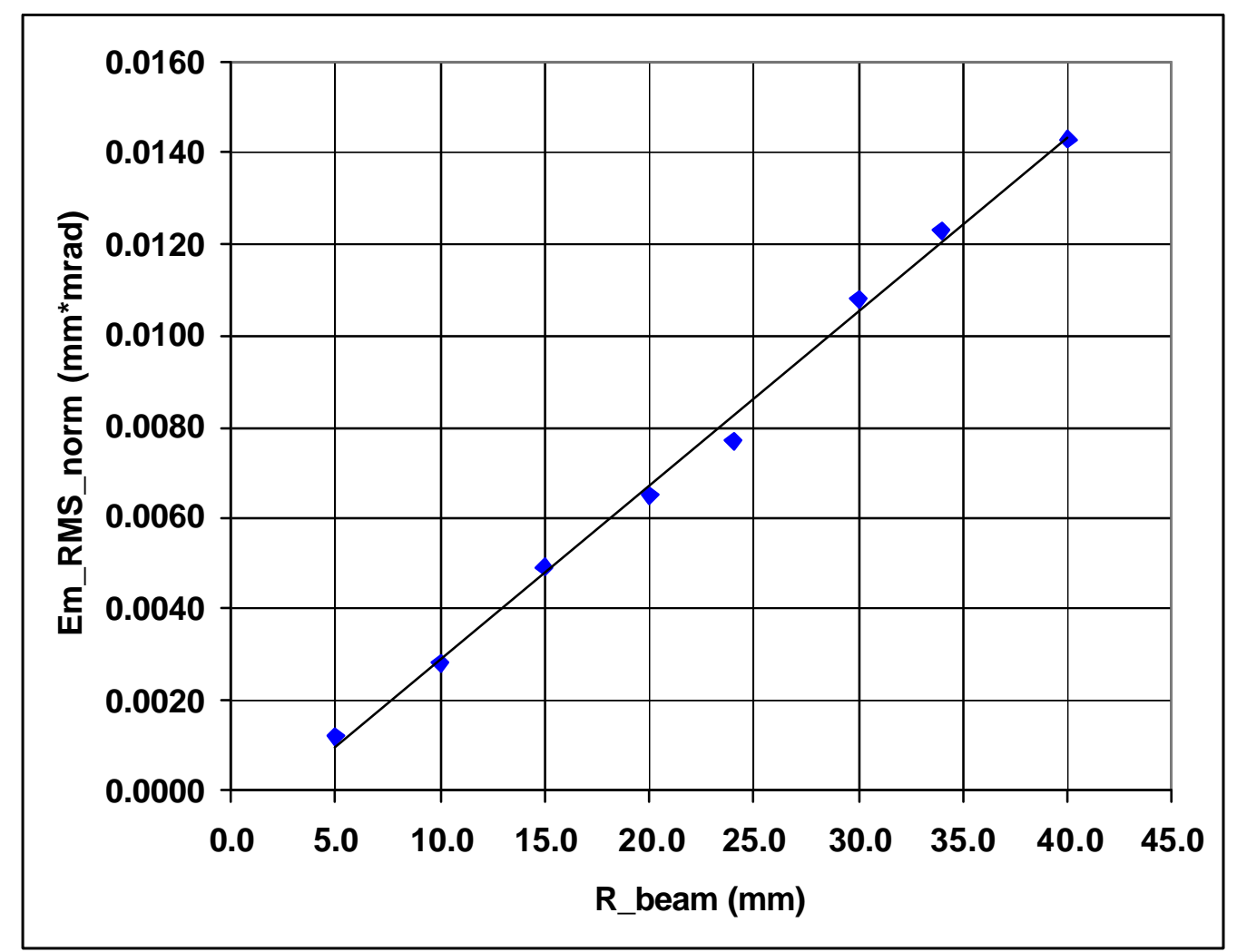

Fig. 5. Dependence of acquired emittance of ion beam in a gridded lens on the radius of ion beam for voltages optimized for focal distance $\mathrm{F}=202 \mathrm{~mm}$. Solid line is a linear fit.

The curves in Fig. 6 allow one to find an optimum combination of voltages on the grid and on the correcting electrode for a desired focal distance with minimum spherical aberrations for a given geometry of the lens. 


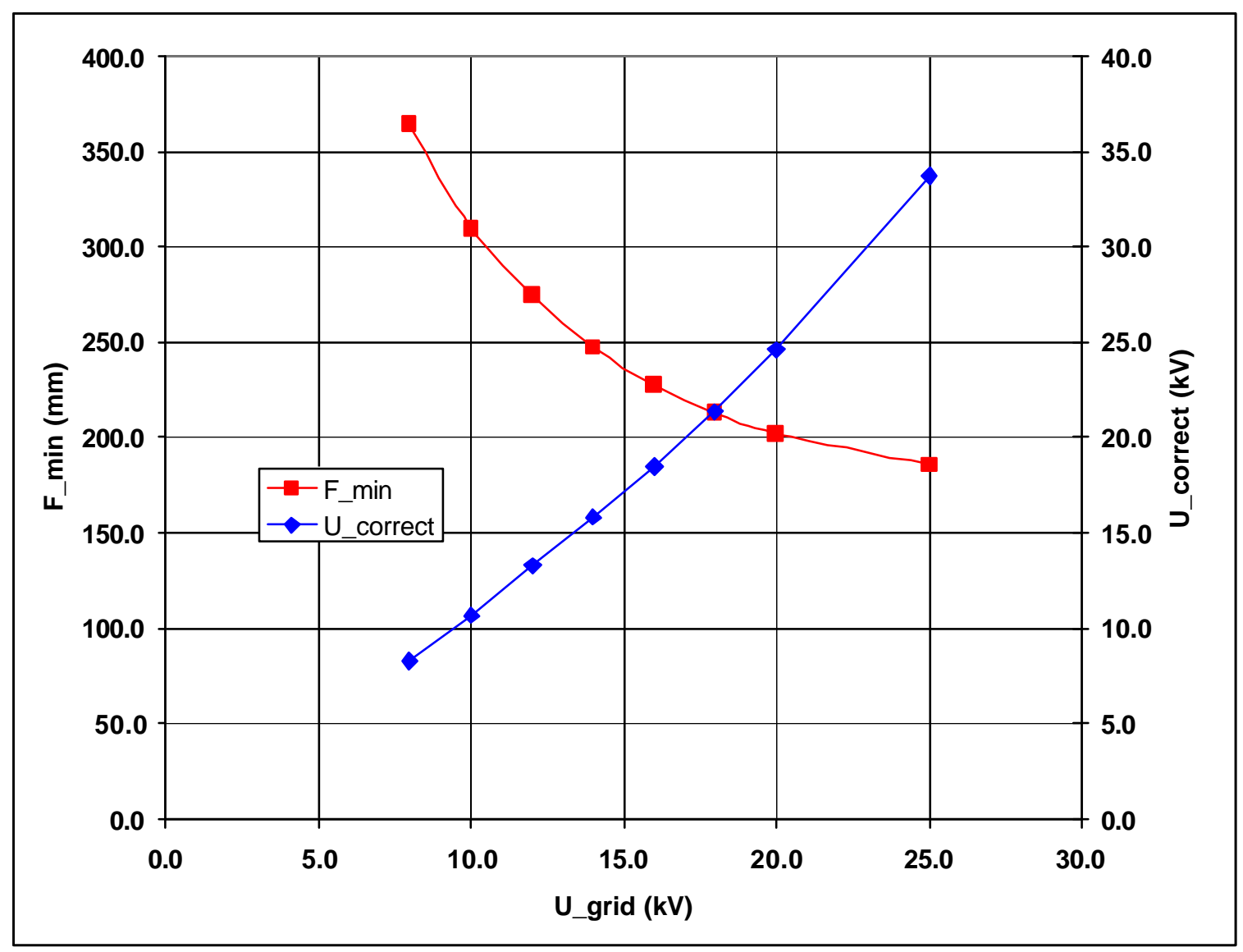

Fig. 6. Dependences of voltage on the correcting electrode $U_{\text {correct }}$ and corresponding focal distance $F_{\min }$ of the lens on the grid voltage $U_{\text {grid }}$ for focusing with minimum aberrations.

Beam radius $r_{\text {beam }}=40 \mathrm{~mm}$, ion energy $E_{\text {ion }}=17.5 \mathrm{keV} /$ charge.

The performances of different types of electrostatic lenses with the same ID of $104 \mathrm{~mm}$ are presented in Fig. 7 for identical parameters of ion beam. This graph demonstrates that the lens with isolated electrodes retains low aberrations over a wide range of focal distances. The gridded lens with rings at the same potential as the grid may have advantage over the lens with isolated correcting electrodes in a certain range of focal length it is optimized for. For the optimum focusing a unipotential lens with $\mathrm{h}=30 \mathrm{~mm}$ has some lower aberration than the lens with isolated correcting electrode and $\mathrm{h}=10 \mathrm{~mm}$. The beam emittance growth after the accelerating Einzel lens is much higher than any of presented gridded lenses. 


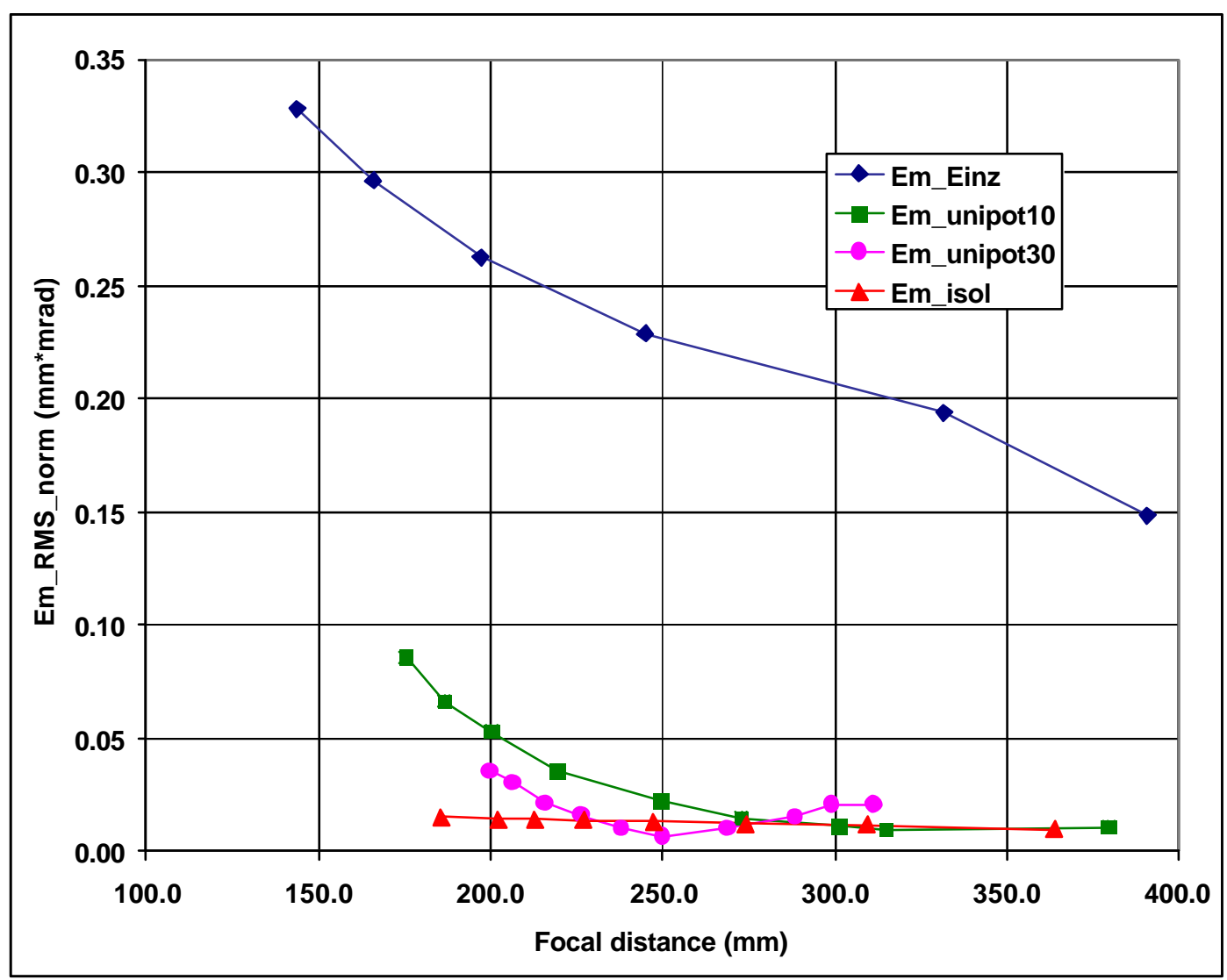

Fig. 7. Dependence of the beam emittance growth in the lens with the same aperture of 104 $\mathrm{mm}$ on the focal distance for accelerating Einzel lens, unipotential gridded lenses width $\mathrm{h}=10 \mathrm{~mm}$ and $\mathrm{h}=30 \mathrm{~mm}$, and for gridded lens with isolated correcting electrodes $(\mathrm{h}=10 \mathrm{~mm})$ optimized for minimum aberrations.

\section{Conclusion.}

A method of correcting the spherical aberration of the 1-grid gridded lens by increasing the length of the rings on the radial periphery of the grid is effective for lens application with fixed focal distance.

For applications requiring variable focusing strength a gridded lens with isolated electrodes has an advantage. It allows maintaining low growth of beam emittance in much wider range of focal length.

\section{References}

${ }^{1}$ J. W. Johnson, IEEE Transactions on Nuclear Science, V. 12, No. 3 (765-768) 1965

${ }^{2}$ M. Kato, Journal of Vac. Sci. \& Techn. A, V. 13, N0. 4 (2255-2260) 1995

${ }^{3}$ W. D. Meisburger and E. H. Jacobsen, Optik, 62, No. 4 (359-366)1982

${ }^{4}$ http://www.fieldp.com/ 